\title{
Auszug aus dem \\ Protokoll der Vorstandssitzung
}

\author{
vom 14 . Oktober 1908 .
}

Anwesend die HHrn. Vorstandsmitglieder: W. Nernst, E. Fischer, S. Gabriel, J. F. Holtz, G. Kraemer, C. Liebermann, F. Mylius, A. Pinner, H. Wichelhaus, W. Will, sowie der Generalsekretär Hr. P. Jacobson und der Verwaltungssekretär Hr. R. Stelzuer.

A uszug aus Nr. 20 und 53. Der Vorstand beschließt, daß für don Bezug des "Chemischen Zentralblatts vom Jahrgang 1909 ab bis auf weiteres die folgenden Preise gelten sollen:

Jahresabonnementspreis für Mitglieder innerbalb des deutsch-österreich. Postgebiets . . . . $.40 \mathrm{Mk}$.

Jahresabonnementspreis für Mitglieder außerhalb des deutsch-österreich. Postgebiets . . . . . . 46 »

Jahresabounementspreis für diejenigen deutschen Institute, die zum Bezug für den ermäßigten Preis berechtigt sind . . . . . . . . . . 45 »

Ladenpreis für Abonnement im Bucbbandel . . . 80 »

Diese Erhöhung wird notwendig, da das Budget des »Chemischen Zentralblatts" in den Jahren 1907. und 1908 außerordentliche Zuschüsse aus dem Vermögen der Gesellschaft (1907: 9600 Mk., 1908: schätzungsweise $15000 \mathrm{Mk}$.) erfordert hat, bedingt durch das Anwachsen des Umfangs und besonders durch die Steigerung der Preise fur die Herstellung des Blattes. Trotz dieser Erhöhung wird (bei Voraussetzung eines Jabresumfangs von 300-310 Bogen) der Bogenpreis für Mitglieder im Inlande nur $13 \mathrm{Pfg}$. betragen gegen $14 \mathrm{Pfg}$. im Jahre 1897. Der Lieferungspreis pro Bogen hat daher in dem Zeitraum seit der Übernahme des Blattes durch unsere Gesellschaft eine Verteuerung nicht erfahren, obwobl bekanntlicb in diesem Zeitraum die Preise für Satz und Druck zweimal (1901 und 1906) beträchtlich gesteigert worden sind.

Durch die Erhöbung wird mutmaßlich das Gleichgewicht im Budget des Zentralblatts wieder hergestellt werden, ohne daß indes mit einem Überschuß zu rechnen ist. Bei Beibehaltung des bisherigen Abonnementspreises wäre ein Ausgleich nur möglich, wenn man die Vollständigkeit unseres Referiernrgans durch Beschränkung des zu referierenden Materials beeinträchtigen oder die Referate, welche jetzt die Aufgabe baben, den wesentlichen lnhalt der Originalabbandlung 
zu erschöpfen, durch kurze Anzeigen ersetzen würde. Der Vorstand glaubt im Sinne der Abonnenten zu bandeln, wenn er beide Wege verwirft und dem Zentralblatt seinen bisherigen Charakter erbält.

55. Der Vorstand nimmt davon Kenntnis, da $\beta$ die folgenden Anteilhaber der Gesellschaft $H_{0}$ ofmannhaus m. b. H. auf die Rückzahlung ihrer (ieschäftsanteile verzichtet haben:

Geb. Rat Prof. Dr. C. Liebermann (Berlin) $5000 \mathrm{Mk}$. (zweite Hälfte seines Anteils von $10000 \mathrm{Mk}$.).

Prof. Dr. L. Darmstaedter (Berlin) $5000 \mathrm{Mk}$.

Der Präsident hat bereits im Namen des Vorstandes den beiden Herren den wärmsten Dank der Gesellschaft für ihre Förderung dẹs Hofmanhaus-Unternehmens ausgesprochen.

Auszug aus Nr. 29 und 56. Der Vorstand hat am 19. Mai d. J. beschlossen, daß aus dem Vermögen der Jeutschen Chemischen Gesellschaft ein Betrag von höchstens $50000 \mathrm{Mk}$. für die Rückzahlung von Geschäftsanteilen an die Anteilhaber der Hofmannhaus-Gesellschaft bereitgestellt werden soll, sofern hierdurch die Auflösung der Hofmannhaus-Gesellschaft und der endgültige Übergang des Hofmannhauses in den Besitz der Deutschen Chemischen Gesellschaft ermöglicht wird. Durch die im Protokoll unter Nr. 32 (vergl. diese Berichte 41, 1783 [1908]) und unter Nr. 55 (s. oben) mitgeteilten Verzichtleistungen und Zuwendungen wird die Möglichkeit geschaffen, daß mit einem Beitrage der Dentschen Chemischen Gesellschaft von ca. $49000 \mathrm{Mk}$. - zusammen mit den der Hofmannhaus-Gesellschaft zur Verfügung stehenden Mitteln - denjenigen Gesellschaftern, welchen die Hofmannhaus-Gesellschaft noch zur Rückzahlung ihrer Anteile verpflichtet ist, das seinerzeit von ihnen eingezahlte Kapital zurüčkerstattet werden kann. Die formelle Auflösung der Hofmannhaus-Gesellschaft ist demnach für anfangs November d. J. in Aussicht genommen.

Auszug aus Nr. 31 und 57. Der Vorstand bestimmt, dals nach dem Auszuge der "Berufsgenossenschaft der Chemischen Industrie» aus dem III. und IV. Stockwerk des Hofmannbauses diese beiden Stockwerke - mit Ausnahme von zwei Zimmern im dritten und einem Zimmer im vierten Stockwerk - zu einer Beamtenwohnung umgestaltet werden sollen, bewilligt für die Herrichtung dieser Wohnung (Reparaturen, bauliche Änderungen, Bauleitung) einen Höchstbetrag von $9250 \mathrm{Mk}$., und genehmigt einen Vertrag, nach welchem diese Wohnung dem Generalsekretär Hrn. Prof. Dr. Paul Jacobson vom 1. April 1909 ab vermietet wird. 
Auszug aus Nr. 58. Der Vorstand genehmigt einen Vertrag, nach welchem der Bureaubeante $\mathrm{Hr}$. Bernbard Christoph als Bureaurorsteher für Geschäftsstelle und Bibliothek der Deutschen Chemischen Gesellschaft vom 1. Oktober 1908 ab angestellt wird.

Auszug aus Nr. 60. Der Vorstand bestimmt im Hinblick auf den bevorstehenden Übergang des Hofmannhauses in die Verwaltung der Deutschen Chemischen Gesellschaft die Einsetzung einer Hauskommission; sie soll aus 3 Mitgliedern der Deutschen Chemischen Gesellschaft bestehen, von denen mindestens zwei den jeweiligen Vorstande angehören müssen.

Für den Rest des Jahres 1908 werden die HHrn. G. Kraemer, R. Pschorr und W. Will zu Mitgliedern dieser Kommission gewählt.
Der Vorsitzende:
Der Schriftführer:
W. Nernst.
W. Will.

\section{Mitteilungen.}

\section{Gustav Heller und Kurt Schülke: Über die Friedel-Craftssche Reaktion.}

[Mitteilung aus dem Laboratorium für Angewandte Chemie von $\mathrm{E}$ Beckm an n, Leipzig.]

(Eingegangen am 6. August 1908.)

'Vor einiger Zeit hat G. Heller ${ }^{1}$ ) gezeigt, daß bei der FriedelCraftsschen Reaktion zur Darstellung von o-Ketonsäuren das angewandte Aluminiumchlorid nicht katalytisch wirkt, sondern daß zur quantitativen Durchführung der Umsetzung genau ein Molekül $\mathrm{Al}_{2} \mathrm{Cl}_{6}$ auf ein Molekül Phthalsäureanbydrid verbraucht wird, und es ließen sich so $97 \%$ der theoretischen Menge an Benzoylbenzoesäure erbalten.

Was nun den Mechanismus der Reaktion anbelangt, so kann man beobachten, daß bei der Umsetzung allmählich Anhydrid und Aluminiumchlorid in Lösung gehen; es spaltet sich Salzsäure ab, und scbließlich erbält man eine zäbe Masse, die nicht krystallisiert und durch Wasser in ein Aluminiumsalz der Benzoylbenzoesäure überge-

1) Ztschr. für angew. Chem. 19, 669 [1906]. 